\title{
Solvability of Third-Order Three-Point Boundary Value Problems
}

\author{
Dongyuan Liu and Zigen Ouyang \\ School of Mathematics and Physics, University of South China, Hengyang 421001, China \\ Correspondence should be addressed to Zigen Ouyang; zigenouyang@163.com
}

Received 28 October 2013; Accepted 9 April 2014; Published 29 April 2014

Academic Editor: Patricia J. Y. Wong

Copyright (C) 2014 D. Liu and Z. Ouyang. This is an open access article distributed under the Creative Commons Attribution License, which permits unrestricted use, distribution, and reproduction in any medium, provided the original work is properly cited.

We are interested in the existence theorems for a third-order three-point boundary value problem. In the nonresonant case, using the Krasnosel'skii fixed point theorem, we obtain some sufficient conditions for the existence of the positive solutions. In addition, we focus on the resonant case, the boundary value problem being transformed into an integral equation with an undetermined parameter, and the existence conditions being obtained by the Intermediate Value Theorem.

\section{Introduction}

Consider the following third-order nonlinear differential equations:

$$
y^{\prime \prime \prime}(t)+f(t, y(t))=0, \quad 0 \leq t \leq 1,
$$

subject to the following boundary value conditions:

$$
y^{\prime}(0)=0, \quad y^{\prime \prime}(0)=0, \quad y(1)=\alpha y(\eta),
$$

where $\eta \in(0,1), 0 \leq \alpha \leq 1$, and $f(t, u) \in C([0,1] \times R)$.

Recently, the existence of solutions for boundary value problem has been investigated by many authors [1-9]. Further, many authors focused on the existence of solutions or positive solutions for higher order differential equations with boundary value problems [10-16].

Sun [15] has investigated the following three-order threepoint boundary value problem:

$$
y^{\prime \prime \prime}(t)-\lambda a(t) f(t, y(t))=0, \quad 0 \leq t \leq 1,
$$

with

$$
y(0)=y^{\prime \prime}(1)=y^{\prime}(\eta)=0,
$$

where $\eta \in[1 / 2,1), \lambda$ is a positive parameter, $a(t) \in C^{+}[0,1]$, and $f \in C([0,1] \times[0, \infty))$. Using the Krasnosel'skii fixed point theorem, they obtained some existence conditions for the positive solutions of the problem (3)-(4).

Usually, by constructing Green's function $\bar{G}(t, s)$, the authors transform the problem (1)-(2) $(0<\alpha<1)$ into the following integral equation:

$$
y(t)=\int_{0}^{1} \bar{G}(t, s) f(s, y(s)) d s, \quad 0 \leq t \leq 1 .
$$

Therefore, some fixed point theorems can be used to prove the existence of the positive solutions of the problem (1)-(2).

However, if we consider the problem (1)-(2) when $\alpha=$ 1 , the respective integral equation of the problem (1)-(2) has not the form of (5). Thus, we cannot prove the existence of solutions of (1)-(2) only by fixed point theorems.

Despite the success in the study of (1)-(2) and (3)-(4), it has been recognized that for the resonant cases, that is, (1)(2) with $\alpha=1$, no much work has been known except recent work $[12,17,18]$ and the references therein where the so-called coincidence-degree method was employed.

$\mathrm{Lu}$ and $\mathrm{Ge}[12]$ considered the following higher-order $m$ point boundary value problem:

$$
\begin{array}{r}
y^{(k)}(t)=F\left(t, y(t), y^{\prime}(t), \ldots, y^{(k-1)}(t)\right)+e(t), \\
0 \leq t \leq 1,
\end{array}
$$


with

$$
\begin{gathered}
y^{\prime}(0)=y^{\prime \prime}(0)=\cdots=y^{(k-1)}(0)=0, \\
y(1)=\sum_{i=1}^{m-2} \alpha_{i} y\left(\eta_{i}\right),
\end{gathered}
$$

where $0<\eta_{1}<\eta_{2}<\cdots<\eta_{m-2}$ and $\sum_{i=1}^{m-2} \alpha_{i}=1$. Using the coincidence-degree method, they obtained a sufficient condition for the existence of solutions for the problem (6)(7).

More recently, Ouyang and $\mathrm{Li}$ [19] have discussed a class of fractional order differential equations of the following three-point boundary value problem with resonance:

$$
\begin{gathered}
y^{\alpha}(t)+f(t, y(t))=0, \quad 0 \leq t \leq 1, \quad 1<\alpha \leq 2, \\
y(0)=0, \quad y(1)=\frac{1}{\eta^{\alpha-1}} y(\eta) .
\end{gathered}
$$

Using a new method, they obtained some sufficient conditions for the existence of solutions for the fractional order boundary value problem (8).

The purpose of this paper is to study the problem (1)(2) for the nonresonant case and the resonant case. In the nonresonant case, the Krasnosel'skii fixed point theorem is used to prove the existence of positive solutions for the problem (1)-(2). In the resonant case, a completely new method is incorporated; we transform the problem into an integral equation with an undetermined parameter. The Intermediate Value Theorem is applied to determine the particular value of the parameter so that true solutions exist. Not only the existence conditions of the solutions, but also the prove of the main results are more simple than [12].

We introduce two lemmas as follows.

Lemma 1 (the Krasnosel'skii fixed point theorem [20]). Let $P$ be a cone in a Banach space $X$. Assume that $\Omega_{1}$ and $\Omega_{2}$ are open subsets of $X$ with $0 \in \Omega_{1}$ and $\bar{\Omega}_{1} \subseteq \Omega_{2}$. Suppose that $T$ : $P \cap\left(\bar{\Omega}_{2} \backslash \Omega_{1}\right) \rightarrow P$ is a completely continuous operator so that either

(i) $\|T u\| \leq\|u\|$ for $u \in P \cap \partial \Omega_{1}$ and $\|T u\| \geq\|u\|$ for $u \in P \cap \partial \Omega_{2}$

or

(ii) $\|T u\| \geq\|u\|$ for $u \in P \cap \partial \Omega_{1}$ and $\|T u\| \leq\|u\|$ for $u \epsilon$ $P \cap \partial \Omega_{2}$. Then $T$ has a fixed point $u \in P \cap\left(\bar{\Omega}_{2} \backslash \Omega_{1}\right)$.

Lemma 2 (see [21]). Let $C$ be a closed and convex subset of a Banach space $X$. Assume that $U$ is a relatively open subset of $C$ with $0 \in U$ and $T: \bar{U} \rightarrow C$ is completely continuous. Then at least one of the following two properties holds:

(i) $T$ has a fixed point in $\bar{U}$; (ii) there exist $u \in \partial U$ and $\lambda \in(0,1)$ with $u=\lambda T u$.

The paper is arranged as follows. In Section 2, we discuss the existence of the positive solutions of the problem (1)-(2) in nonresonant case. Section 3 is devoted to the existence of the solutions of the problem (1)-(2) in resonant case. Finally, we give some examples to illustrate our results.
For convenience, we set

$$
g_{0}=\lim _{u \rightarrow 0^{+}} \frac{g(u)}{u}, \quad g_{\infty}=\lim _{u \rightarrow \infty} \frac{g(u)}{u} .
$$

To present our result, we assume that

$\left(\mathrm{H}_{1}\right) f(t, u)=a(t) g(u), a(t) \in C^{+}[0,1], g \in C^{+}[0, \infty)$, and $g_{0}=0, g_{\infty}=\infty$, or $g_{0}=\infty, g_{\infty}=0 ;$

$\left(\mathrm{H}_{2}\right) f \in C([0,1] \times R)$; there exist nonnegative functions $m(t), n(t) \in C([0,1])$ so that

$$
|f(t, u)| \leq n(t)|u|^{p}+m(t)
$$

where $0 \leq p \leq 1$

$\left(\mathrm{H}_{3}\right)$ for any $t \in(0,1), f(t, u) \rightarrow \infty$ as $u \rightarrow \infty$, and $f(t, u) \rightarrow-\infty$ as $u \rightarrow-\infty$.

\section{The Nonresonant Case $(\alpha<1)$}

Throughout this paper, we suppose that $C[0,1]$ is a space of continuous functions in $[0,1]$, equipped with the norm

$$
\|y\|=\sup _{0 \leq t \leq 1}|y(t)|, \quad y(t) \in C[0,1] .
$$

In this section, we consider the nonresonant case, that is, (1) with the boundary value problem (2) with $0 \leq \alpha<1$. We have the following theorem.

Theorem 3. Assume that $\left(H_{1}\right)$ holds and $0 \leq \alpha<1$. Then problem (1)-(2) has at least one positive solution.

Proof of Theorem 3. Suppose that $y(t)$ is a solution of the problem (1)-(2). Integrating both sides of (1) three times from 0 to $t$, one gets

$$
\begin{aligned}
y(t) & =-\int_{0}^{t} \int_{0}^{s} \int_{0}^{w} a(v) g(y(v)) d v d w d s+c_{1}+c_{2} t+c_{3} t^{2} \\
& =-\frac{1}{2} \int_{0}^{t}(t-s)^{2} a(s) g(y(s)) d s+c_{1}+c_{2} t+c_{3} t^{2} .
\end{aligned}
$$

Imposing the first two boundary conditions in (2), we have

$$
c_{2}=c_{3}=0 \text {. }
$$

Imposing the last boundary condition in (2), we obtain

$$
\begin{aligned}
c_{1}=\frac{1}{2(1-\alpha)}[ & \int_{0}^{1}(1-s)^{2} a(s) g(y(s)) d s \\
& \left.-\int_{0}^{\eta} \alpha(\eta-s)^{2} a(s) g(y(s)) d s\right] .
\end{aligned}
$$

Substituting (13) and (14) into (12), the problem (1)-(2) is equivalent to the following integral equation:

$$
y(t)=\int_{0}^{1} G(t, s) a(s) g(y(s)) d s,
$$


where $G(t, s)$ is defined by

$$
\begin{aligned}
& G(t, s) \\
& =\frac{1}{2(1-\alpha)} \\
& \quad \times \begin{cases}(1-s)^{2}-\alpha(\eta-s)^{2} & \\
-(1-\alpha)(t-s)^{2}, & 0 \leq s \leq \min \{t, \eta\} \leq 1, \\
(1-s)^{2}-\alpha(\eta-s)^{2}, & 0 \leq t \leq s \leq \eta \leq 1, \\
(1-s)^{2}-(1-\alpha)(t-s)^{2}, & 0 \leq \eta \leq s \leq t \leq 1, \\
(1-s)^{2}, & 0 \leq \max \{t, \eta\} \leq s \leq 1 .\end{cases}
\end{aligned}
$$

When $t \geq s$, the function $G(t, s)$ is decreasing with respect to $t$, and $G(t, s)$ is independent on the parameter $t$ when $t<s$. So Green's function $G(t, s)$ satisfies that $0 \leq G(t, s) \leq$ $G(s, s)$. Further, for any $0<\theta<1 / 2$, Green's function $G(t, s)$ satisfies

$$
G(\theta, s) \geq G(1-\theta, s),
$$

and the solution $y(t)$ of (1)-(2) is nonnegative and satisfies

$$
\min _{t \in[\theta, 1-\theta]} y(t) \geq \theta\|y\|
$$

for any $a \in C^{+}[0,1]$ and $g \in C^{+}[0, \infty)$.

In fact, since $G(t, s) \geq 0$, from $\left(\mathrm{H}_{1}\right)$ and (15), $y(t) \in$ $C^{+}[0,1]$. Noticing that

$$
y^{\prime \prime \prime}(t)=-a(t) g(y(t)) \leq 0
$$

thus, $y^{\prime \prime}(t)$ is decreasing; it follows from the boundary value condition $y^{\prime \prime}(0)=0$ that

$$
y^{\prime \prime}(t) \leq 0, \quad t \in[0,1] .
$$

Hence $y(t)$ is concave; that is, for any $t_{1}, t_{2} \in[0,1]$ and $\gamma \in$ $[0,1]$,

$$
y\left(\gamma t_{1}+(1-\gamma) t_{2}\right) \geq \gamma y\left(t_{1}\right)+(1-\gamma) y\left(t_{2}\right), \quad t \in[0,1] .
$$

Noticing that $y^{\prime}(0)=0$, then $y^{\prime}(t) \leq 0$, and $y(t)$ is nonincreasing; thus $t=0$ is the maximum point of $y(t)$ in $[0,1]$; then $\|y\|=y(0)$. For any $t \in[\theta, 1-\theta]$, one gets

$$
\begin{aligned}
y(t) & \geq y(1-\theta)=y(\theta \times 0+(1-\theta) \times 1) \\
& \geq \theta y(0)+(1-\theta) y(1) \geq \theta y(0)=\theta\|y\| .
\end{aligned}
$$

Therefore, (18) holds.

Suppose that $\theta$ is a given number and satisfies $0<\theta<$ $1 / 2$. We denote a cone $K$ as follows:

$$
K=\left\{y \in C^{+}[0,1] \mid \min _{t \in[\theta, 1-\theta]} y(t) \geq \theta\|y\|\right\},
$$

and define an operator $T$ on $K$ by

$$
T y(t)=\int_{0}^{1} G(t, s) a(s) g(y(s)) d s
$$

Now, we prove the existence of the positive solution of (1)-(2). For convenience, we only prove the case that $g_{0}=0$, $g_{\infty}=\infty$, the prove of the case that $g_{0}=\infty, g_{\infty}=0$ is similar, and we omit it here.

Since $g_{0}=0$, then for $\varepsilon=1 / \int_{0}^{1} G(s, s) a(s) d s>0$, there exists a $r_{1}>0$ such that

$$
g(y) \leq \varepsilon y
$$

for any $0 \leq y \leq r_{1}$. We define the set $K_{1}$ by

$$
K_{1}=\left\{y \in C^{+}[0,1] \mid\|y\| \leq r_{1}\right\} .
$$

Then, for any $y \in \partial K_{1} \cap K$, we have

$$
\begin{aligned}
T y(t) & =\int_{0}^{1} G(t, s) a(s) g(y(s)) d s \\
& \leq \int_{0}^{1} G(s, s) a(s) \varepsilon y(s) d s \\
& \leq \int_{0}^{1} G(s, s) a(s) \varepsilon d s\|y\|=\|y\|=r_{1},
\end{aligned}
$$

which implies that

$$
\|T y(t)\| \leq\|y\|, \quad \text { for } y \in \partial K_{1} \cap K .
$$

From $g_{\infty}=\infty$, for the given $0<\theta<1 / 2$ and

$$
M \geq \frac{1}{\int_{\theta}^{1-\theta} G(1-\theta, s) \theta a(s) d s}>0,
$$

there exists a $r_{2}>0$ so that

$$
g(y) \geq M y \text { for } y \geq r_{2} .
$$

Let

$$
K_{2}=\left\{y \in C^{+}[0,1] \mid\|y\| \leq \frac{1}{\theta} r_{2}\right\} .
$$

Then, for any $y \in \partial K_{2} \cap K$, we obtain from (17) that

$$
\begin{aligned}
\operatorname{Ty}(t) & =\int_{0}^{1} G(t, s) a(s) g(y(s)) d s \\
& \geq \int_{\theta}^{1-\theta} G(1-\theta, s) a(s) M y(s) d s \\
& \geq M \int_{\theta}^{1-\theta} G(1-\theta, s) \theta a(s) d s\|y\| \geq\|y\|=\frac{1}{\theta} r_{2},
\end{aligned}
$$

which follows

$$
\|T y(t)\| \geq\|y\|, \quad \text { for } y \in \partial K_{2} \cap K .
$$

It is easy to show that $T$ is a completely continuous function. Combining (28)-(33) and Lemma 1, the operator $T$ has at least one fixed point in $K \cap\left(\overline{K_{2}} \backslash K_{1}\right)$, which is a positive solution of (15). The proof is completed. 
Suppose that

$\left(\widetilde{\mathrm{H}}_{1}\right): a(t) g(u) \leq f(t, u) \leq b(t) g(u), a(t), b(t) \in C^{+}[0,1]$, $g \in C^{+}[0, \infty)$, and $g_{0}=0, g_{\infty}=\infty$, or $g_{0}=\infty$, $g_{\infty}=0$.

From the proof of Theorem 3, we have the following.

Corollary 4. Assume that $\left(\widetilde{H}_{1}\right)$ holds and $0 \leq \alpha<1$. Then problem (1)-(2) has at least one positive solution.

\section{The Resonant Case $(\alpha=1)$}

In this case, the boundary value condition (2) can be rewritten as

$$
y^{\prime}(0)=0, \quad y^{\prime \prime}(0)=0, \quad y(1)=y(\eta) .
$$

We have the following main theorem.

Theorem 5. Assume that $\left(\mathrm{H}_{2}\right)$ and $\left(\mathrm{H}_{3}\right)$ hold. Further, suppose that

$$
\sup _{t \in[0,1]} \int_{0}^{1} \bar{G}(s, s) n(s) d s<1,
$$

where

$$
\begin{aligned}
& \bar{G}(t, s) \\
& =\frac{1}{2(1-\eta)} \\
& \quad \times \begin{cases}(1-s)^{2}-\eta(\eta-s)^{2} \\
-(1-\eta)(t-s)^{2}, & 0 \leq s \leq \min \{t, \eta\} \leq 1, \\
(1-s)^{2}-\eta(\eta-s)^{2}, & 0 \leq t \leq s \leq \eta \leq 1, \\
(1-s)^{2}-(1-\eta)(t-s)^{2}, & 0 \leq \eta \leq s \leq t \leq 1, \\
(1-s)^{2}, & 0 \leq \max \{t, \eta\} \leq s \leq 1 .\end{cases}
\end{aligned}
$$

Then problem (1)-(34) has at least one solution.

Proof of Theorem 5. Using a similar method as in the proof of Theorem 3, the problem (1)-(34) is equivalent to the following integral equation:

$$
y(t)=\int_{0}^{1} \bar{G}(t, s) f(s, y(s)) d s+y(1),
$$

where $\bar{G}(t, s)$ is defined by (36).

It is obvious that Green's function $\bar{G}(t, s)$ is decreasing with respect to $t$ when $t \geq s$, and it is independent on the parameter $t$ when $t<s$; thus, the function $\bar{G}(t, s)$ is not increasing with respect to $t \in[0,1]$ and $0 \leq \bar{G}(t, s) \leq \bar{G}(s, s)$.

Let

$$
w(t)=y(t)-y(1) .
$$

Given any value $y(1), w(t)$ satisfies the Hammerstein integral equation by (37):

$$
\begin{gathered}
w(t)=\int_{0}^{1} \bar{G}(t, s) f(s, w(s)+y(1)) d s, \\
w(1)=w(\eta)=0 .
\end{gathered}
$$

To obtain the solvability of (39), we replace $y(1)$ by a real constant $\mu$; that is,

$$
\begin{gathered}
w(t)=\int_{0}^{1} \bar{G}(t, s) f(s, w(s)+\mu) d s, \\
w(1)=0 .
\end{gathered}
$$

Define the Hammerstein operator:

$$
T w(t)=\int_{0}^{1} \bar{G}(t, s) f(s, w(s)+\mu) d s, \quad w(t) \in C[0,1]
$$

for any real number $\mu$. From the condition $\left(\mathrm{H}_{2}\right)$, it is easy to know that

$$
\begin{aligned}
|T w(t)| \leq & \int_{0}^{1} \bar{G}(t, s)\left(n(s)|w(s)+\mu|^{p}+m(s)\right) d s \\
\leq & \|w(s)\|^{p} \int_{0}^{1} \bar{G}(s, s) n(s) d s \\
& +\int_{0}^{1} \bar{G}(s, s)\left(|\mu|^{p}+m(s)\right) d s ;
\end{aligned}
$$

here, we have made use of

$$
|a+b|^{p} \leq|a|^{p}+|b|^{p}
$$

for any $0 \leq p \leq 1$. By (35), that is,

$$
\sup _{t \in[0,1]} \int_{0}^{1} \bar{G}(s, s) n(s) d s<1
$$

we then see that the operator $T$ maps the ball $B_{r}$ onto itself, where

$$
\begin{aligned}
B_{r}=\{w \in C & {\left.[0,1]:\|w\|_{\infty} \leq r\right\}, } \\
r=\max \{1, & {\left[1-\int_{0}^{1} \bar{G}(s, s) n(s) d s\right]^{-1} } \\
& \left.\times\left(\int_{0}^{1} \bar{G}(s, s)\left(|\mu|^{p}+m(s)\right) d s\right)\right\} .
\end{aligned}
$$

It is easy to show that the operator $T$ is a compact operator. From Lemma 2 and using a similar method of Theorem 3.6 in [22], we obtain that the operator $T$ has a fixed point $w_{\mu}(t)$ for any real number $\mu$. Let $w_{\mu}$ be the fixed point of $T$ with a given parameter $\mu$; that is, $T w_{\mu}=w_{\mu}$. For the solvability of (40), we need to find a $\mu_{0}$ so that $w_{\mu_{0}}(1)=0$; that is,

$$
w_{\mu_{0}}(1)=\int_{0}^{1} \bar{G}(1, s) f\left(s, w_{\mu_{0}}(s)+\mu_{0}\right) d s=0 .
$$

Define

$$
L(\mu)=w_{\mu}(1)=\int_{0}^{1} \bar{G}(1, s) f\left(s, w_{\mu}(s)+\mu\right) d s .
$$


We only need to claim by assumption $\left(\mathrm{H}_{3}\right)$ that

$$
\lim _{\mu \rightarrow \pm \infty} L(\mu)= \pm \infty
$$

It is obvious that $L(\mu)$ is continuously dependent on the parameter $\mu$; this would help us to show the existence of $\mu_{0}$ such that $L\left(\mu_{0}\right)=0$.

Now, we show that $L(\mu) \rightarrow \infty$ as $\mu \rightarrow \infty$.

On the contrary, assume that there exists a sequence $\left\{\mu_{n}\right\}$ such that

$$
\lim _{n \rightarrow \infty} \mu_{n}=\infty, \quad \lim _{n \rightarrow \infty} L\left(\mu_{n}\right)=l<\infty .
$$

We now claim that for all $t \in[0,1], w_{\mu_{n}}(t)+\mu_{n}$ is unbounded from below, which is dependent on $t$ and $n$. In fact, suppose that $w_{\mu_{n}}(t)+\mu_{n}$ is bounded from below by a constant $-m$; by assumption $\left(\mathrm{H}_{3}\right), f(t, u)$ is bounded from below for $t \in[0,1]$ and $u \in[-m, \infty)$; that is, there exists an $\bar{M}>0$ so that

$$
f(t, u) \geq-\bar{M}, \quad \text { for } t \in[0,1], u \in[-m, \infty) .
$$

Replacing $u$ in (50) by $w_{\mu_{n}}(t)+\mu_{n}$, one gets

$$
f\left(t, w_{\mu_{n}}(t)+\mu_{n}\right) \geq-\bar{M}
$$

for $w_{\mu_{n}}(t)+\mu_{n} \geq-m$ and $t \in[0,1]$, which implies that the fixed point of $T$ satisfies

$$
\begin{aligned}
w_{\mu_{n}}(t) & =\int_{0}^{1} \bar{G}(t, s) f\left(s, w_{\mu_{n}}(s)+\mu_{n}\right) d s \\
& \geq-\bar{M} \int_{0}^{1} \bar{G}(t, s) d s \geq-\bar{M},
\end{aligned}
$$

which implies that $w_{\mu_{n}}(t)+\mu_{n} \rightarrow \infty$ as $\mu_{n} \rightarrow \infty$. From the condition $\left(\mathrm{H}_{3}\right), f\left(t, w_{\mu_{n}}(t)+\mu_{n}\right) \rightarrow \infty$ as $\mu_{n} \rightarrow \infty$ for all $t \in(0,1]$. Noticing that $\bar{G}(\eta, s)=\left((1-s)^{2}-(\eta-s)^{2}\right) / 2(1-\eta)$ for $0 \leq s \leq \eta$, we have

$$
\begin{aligned}
L\left(\mu_{n}\right) & \geq \int_{0}^{\eta} \bar{G}(\eta, s) f\left(s, w_{\mu_{n}}(s)+\mu_{n}\right) d s \\
& =\int_{0}^{\eta} \frac{(1-s)^{2}-(\eta-s)^{2}}{2(1-\eta)} f\left(s, w_{\mu_{n}}(s)+\mu_{n}\right) d s \\
& >\frac{(1-\eta)^{2}-(\eta-\eta)^{2}}{2(1-\eta)} \int_{0}^{\eta} f\left(s, w_{\mu_{n}}(s)+\mu_{n}\right) d s \rightarrow \infty
\end{aligned}
$$

as $\mu_{n} \rightarrow \infty$, which contradicts $\lim _{n \rightarrow \infty} L\left(\mu_{n}\right)<\infty$; thus, our claim is true, and

$$
\lim _{\mu_{n} \rightarrow \infty} \min _{s \in[0,1]} w_{\mu_{n}}(s)=-\infty
$$

Since $w_{\mu_{n}}(t)$ is a fixed point of the operator $T$, that is, the function $w_{\mu_{n}}(t)$ satisfies

$$
w_{\mu_{n}}(t)=\int_{0}^{1} \bar{G}(t, s) f\left(s, w_{\mu_{n}}(s)+\mu_{n}\right) d s .
$$

From (54) and (55), it is impossible that

$$
f\left(t, w_{\mu_{n}}(t)+\mu_{n}\right) \geq 0, \quad \forall t \in[0,1]
$$

as $\mu_{n}$ is sufficiently large.

Now, we define

$$
\begin{aligned}
& \widetilde{I_{n}}=\left\{t \in[0,1]: w_{\mu_{n}}(t)+\mu_{n}>0\right\}, \\
& \widehat{I_{n}}=\left\{t \in[0,1]: w_{\mu_{n}}(t)+\mu_{n} \leq 0\right\} .
\end{aligned}
$$

Then, $\widehat{I_{n}}$ is not empty, and $\widetilde{I_{n}} \cap \widehat{I}_{n}=\phi$. In fact, assume that $\widehat{I}_{n}$ is empty; then, $f\left(t, w_{\mu_{n}}(t)+\mu_{n}\right)$ is bounded from below for any $t \in[0,1]$ and $\mu_{n} \in[0, \infty)$, which follows from (55) and $\left(\mathrm{H}_{3}\right)$ that $w_{\mu_{n}}(t)$ is bounded from below. This contradicts (54).

From (50), there exists a positive number $\widetilde{M}$ so that

$$
f\left(t, w_{\mu_{n}}(t)+\mu_{n}\right) \geq-\widetilde{M}, \quad \text { for } t \in \widetilde{I_{n}}
$$

Notice that $w_{\mu_{n}}(t)<0$ for any $\mu_{n}>0$ and $t \in \widehat{I_{n}}$, and $\lim _{\mu_{n} \rightarrow \infty} w_{\mu_{n}}(t)=-\infty$ for any $t \in \widehat{I_{n}}$.

Therefore, we have from $\bar{G}(t, s) \leq \bar{G}(s, s)$ that

$$
\begin{aligned}
w_{\mu_{n}}(t)= & \int_{0}^{1} \bar{G}(t, s) f\left(s, w_{\mu_{n}}(s)+\mu_{n}\right) d s \\
= & \int_{\widetilde{I}_{n}} \bar{G}(t, s) f\left(s, w_{\mu_{n}}(s)+\mu_{n}\right) d s \\
& +\int_{\widehat{I_{n}}} \bar{G}(t, s) f\left(s, w_{\mu_{n}}(s)+\mu_{n}\right) d s .
\end{aligned}
$$

From $\left(\mathrm{H}_{3}\right)$, we have from (58) and (59) that

$$
\begin{aligned}
w_{\mu_{n}}(t) \geq & -\widetilde{M} \int_{\widetilde{I}_{n}} \bar{G}(s, s) d s-\min _{s \in \widehat{I}_{n}}\left|w_{\mu_{n}}(s)+\mu_{n}\right|^{p} \\
& \times \int_{\widetilde{I_{n}}} \bar{G}(s, s) n(s) d s-\int_{\widehat{I_{n}}} \bar{G}(s, s) m(s) d s \\
\geq & -\widetilde{M} \int_{0}^{1} \bar{G}(s, s) d s-\min _{s \in \widehat{I}_{n}}\left|w_{\mu_{n}}(s)\right|^{p} \\
& \times \int_{0}^{1} \bar{G}(s, s) n(s) d s-\int_{0}^{1} \bar{G}(s, s) m(s) d s \\
\geq & -\widetilde{M} \int_{0}^{1} \bar{G}(s, s) d s-\int_{0}^{1} \bar{G}(s, s) n(s) d s \\
& +\min _{s \in[0,1]} w_{\mu_{n}}(s) \int_{0}^{1} \bar{G}(s, s) m(s) d s .
\end{aligned}
$$

Taking the minimal values of both sides of the above, it yields

$$
\begin{aligned}
\min _{s \in[0,1]} w_{\mu_{n}}(s) \geq & -\left[1-\int_{0}^{1} \bar{G}(s, s) m(s) d s\right]^{-1} \\
& \times\left\{\widetilde{M} \int_{0}^{1} \bar{G}(s, s) d s+\int_{0}^{1} \bar{G}(s, s) n(s) d s\right\} .
\end{aligned}
$$

This contradicts (54). 
Therefore, we have proved that $\lim _{\mu \rightarrow \infty} L(\mu)=\infty$. By a similar method, we can prove that $\lim _{\mu \rightarrow-\infty} L(\mu)=-\infty$ and the detail is omitted.

Since the function $L(\mu)$ is continuous with respect to the parameter $\mu$. From the Intermediate Value Theorem, there exists a $\mu_{0}$ so that $L\left(\mu_{0}\right)=w_{\mu_{0}}(1)=0$.

Let

$$
y(t)=w_{\mu_{0}}(t)+\mu_{0}
$$

Then, $y(1)=\mu_{0}$. From (62) and (39), it is obvious that $y(t)$ solves

$$
\begin{gathered}
y(t)=\int_{0}^{1} \bar{G}(t, s) f(s, y(s)) d s+y(1), \\
y(\eta)=y(1),
\end{gathered}
$$

which implies that $y(t)$ is a solution of the problem (1)-(34). This completes the proof.

\section{Examples}

In this section, we give two examples to illustrate our main result.

Example 1. Consider the following boundary value problem:

$$
\begin{aligned}
& y^{\prime \prime \prime}+(t+1) y^{\gamma}=0, \\
& y^{\prime}(0)=y^{\prime \prime}(0)=0, \quad y(1)=\frac{1}{2} y\left(\frac{1}{2}\right),
\end{aligned}
$$

where

$$
a(t)=(t+1), \quad g(y)=y^{\gamma}
$$

From Theorem 3, the problem (64) has a positive solution if $0<\gamma<1$.

Example 2. Consider the following boundary value problem:

$$
\begin{aligned}
& y^{\prime \prime \prime}+d(t+1) g(y)-\sin t=0, \\
& y^{\prime}(0)=y^{\prime \prime}=0, \quad y(1)=y\left(\frac{1}{2}\right),
\end{aligned}
$$

where

$$
g(y)= \begin{cases}\frac{y^{3}}{1+y^{2}}, & |y| \leq 1 \\ \frac{1}{2} y, & |y|>1\end{cases}
$$

Here $f(t, y)=d(t+1) g(y)-\sin t$. Thus, $|f(t, y)| \leq n(t)|y|+$ $m(t)$ with

$$
n(t)=\frac{1}{2} d(t+1), \quad m(t)=|\sin t| .
$$

By applying our theorems, the problem (66) has a solution if $d<256 / 49$.

\section{Conflict of Interests}

The authors declare that there is no conflict of interests regarding the publication of this paper.

\section{Acknowledgments}

The work was supported by the NSF of Hunan Province (no. 13JJ3074), Major Project of Department of Education of Hunan Province (no. 13A088), the Scientific Research Foundation of Hengyang City (no. J1), and the Construct Program in USC.

\section{References}

[1] R. P. Agarwal, M. Meehan, and D. O'Regan, Fixed Point Theorey and Applictions, Cambridge Unicersity Press, Cambridge, UK, 2001.

[2] M. K. Kwong, "The shooting method and multiple solutions of two/multi-point BVPs of second-order ODE," Electronic Journal of Qualitative Theory of Differential Equations, vol. 6, pp. 1-14, 2006.

[3] M. K. Kwong and J. S. W. Wong, "An optimal existence theorem for positive solutions of a four-point boundary value problem," Electronic Journal of Differential Equations, no. 165, 8 pages, 2009.

[4] M. K. Kwong and J. S. W. Wong, "The shooting method and nonhomogeneous multipoint BVPs of second-order ODE," Boundary Value Problems, vol. 2007, Article ID 64012, 16 pages, 2007.

[5] M. K. Kwong and J. S. W. Wong, "Solvability of second-order nonlinear three-point boundary value problems," Nonlinear Analysis. Theory, Methods \& Applications A: Theory and Methods, vol. 73, no. 8, pp. 2343-2352, 2010.

[6] B. Liu, "Positive solutions of a nonlinear three-point boundary value problem," Applied Mathematics and Computation, vol.132, no. 1, pp. 11-28, 2002.

[7] B. Liu, "Positive solutions of a nonlinear three-point boundary value problem," Computers \& Mathematics with Applications, vol. 44, no. 1-2, pp. 201-211, 2002.

[8] Y. P. Sun and L. Liu, "Solvability for a nonlinear second-order three-point boundary value problem," Journal of Mathematical Analysis and Applications, vol. 296, no. 1, pp. 265-275, 2004.

[9] Z. Zhang and J. Wang, "Positive solutions to a second order three-point boundary value problem," Journal of Mathematical Analysis and Applications, vol. 285, no. 1, pp. 237-249, 2003.

[10] L. Kong and Q. Kong, "Higher order boundary value problems with nonhomogeneous boundary conditions," Nonlinear Analysis. Theory, Methods \& Applications A: Theory and Methods, vol. 72, no. 1, pp. 240-261, 2010.

[11] H. Li and Y. Liu, "Multiplicity of solutions for some fourthorder m-point boundary-value problems," Electronic Journal of Differential Equations, vol. 2010, Article ID 749059, 9 pages, 2010.

[12] S. P. Lu and W. G. Ge, "On the existence of $m$-point boundary value problem at resonance for higher order differential equation," Journal of Mathematical Analysis and Applications, vol. 287, no. 2, pp. 522-539, 2003.

[13] H.-R. Pei and J.-P. Sun, "Positive solution for fourth-order $m$ point nonhomogeneous boundary value problems," Journal of 
Applied Mathematics and Computing, vol. 32, no. 2, pp. 443-452, 2010.

[14] J.-P. Sun and Q.-Y. Ren, "Existence of solution for third-order $m$ point boundary value problem," Applied Mathematics E-Notes, vol. 10, pp. 268-274, 2010.

[15] Y. P. Sun, "Positive solutions of singular third-order three-point boundary value problem," Journal of Mathematical Analysis and Applications, vol. 306, no. 2, pp. 589-603, 2005.

[16] X. M. Zhang, M. Q. Feng, and W. G. Ge, "Existence results for nonlinear boundary-value problems with integral boundary conditions in Banach spaces," Nonlinear Analysis. Theory, Methods \& Applications A: Theory and Methods, vol. 69, no. 10, pp. 3310-3321, 2008.

[17] W. Feng and J. R. L. Webb, "Solvability of $m$-point boundary value problems with nonlinear growth," Journal of Mathematical Analysis and Applications, vol. 212, no. 2, pp. 467-480, 1997.

[18] X. L. Han, "Positive solutions for a three-point boundary value problem at resonance," Journal of Mathematical Analysis and Applications, vol. 336, no. 1, pp. 556-568, 2007.

[19] Z. G. Ouyang and G. Z. Li, "Existence of solutions for a class of nonlinear fractional order three-point boundary value problem with resonance," Boundary Value Problems, vol. 2012, p. 68, 2012.

[20] M. A. Krasnosel'skii, Topological Methods in the Theory of Nonlinear Integral Equations, The Macmillan Co, New York, NY, USA, 1964, Translated by A. H. Armstrong.

[21] A. Granas, R. B. Guenther, and J. W. Lee, "Some general existence principles in the Carathéodory theory of nonlinear differential systems," Journal de Mathématiques Pures et Appliquées. Neuvième Série, vol. 70, no. 2, pp. 153-196, 1991.

[22] Z. G. Ouyang, Y. M. Chen, and S. L. Zou, "Existence of positive solutions to a boundary value problem for a delayed nonlinear fractional differential system," Boundary Value Problems, vol. 2011, Article ID 475126, 17 pages, 2011. 


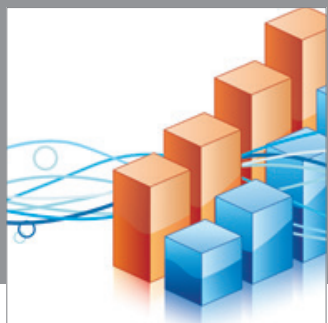

Advances in

Operations Research

mansans

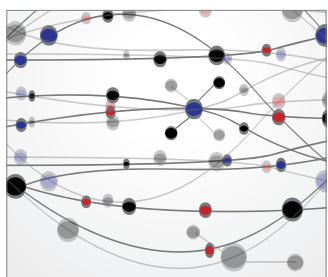

The Scientific World Journal
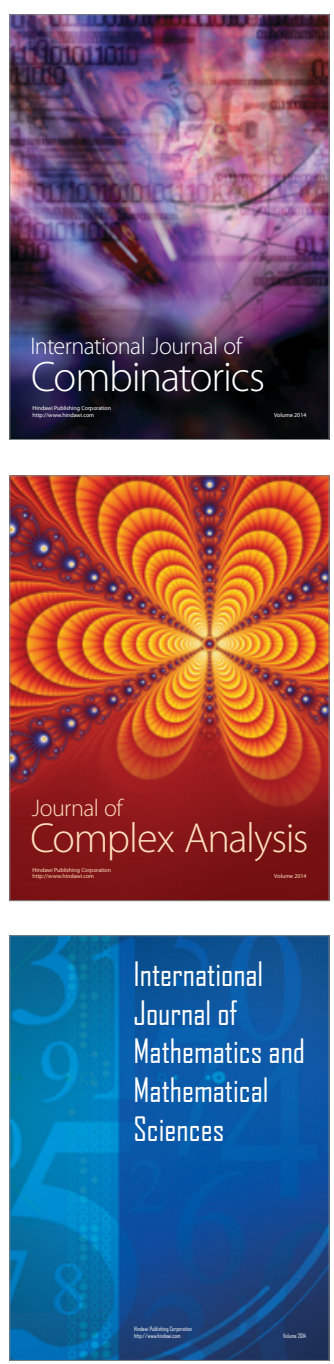
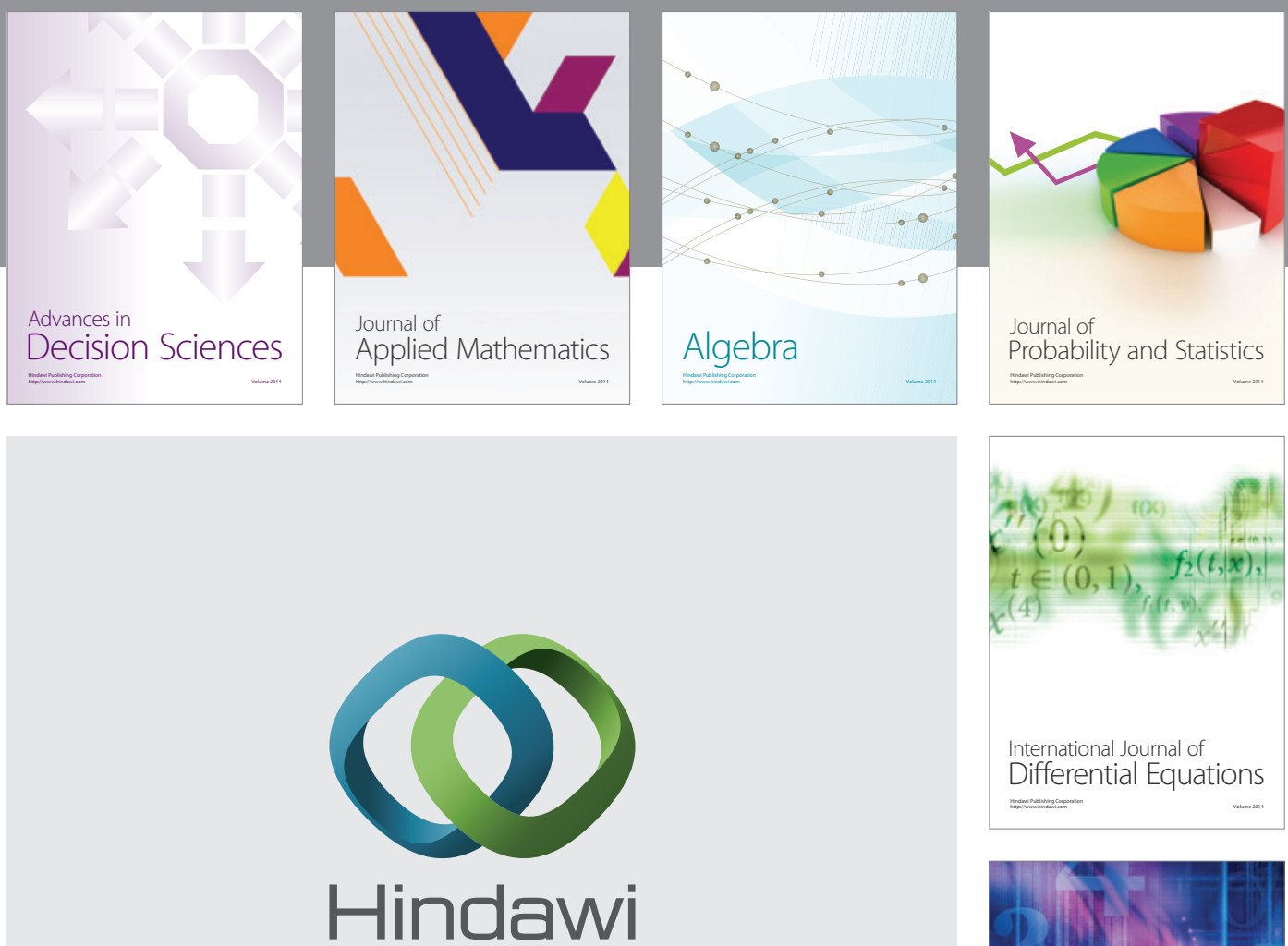

Submit your manuscripts at http://www.hindawi.com
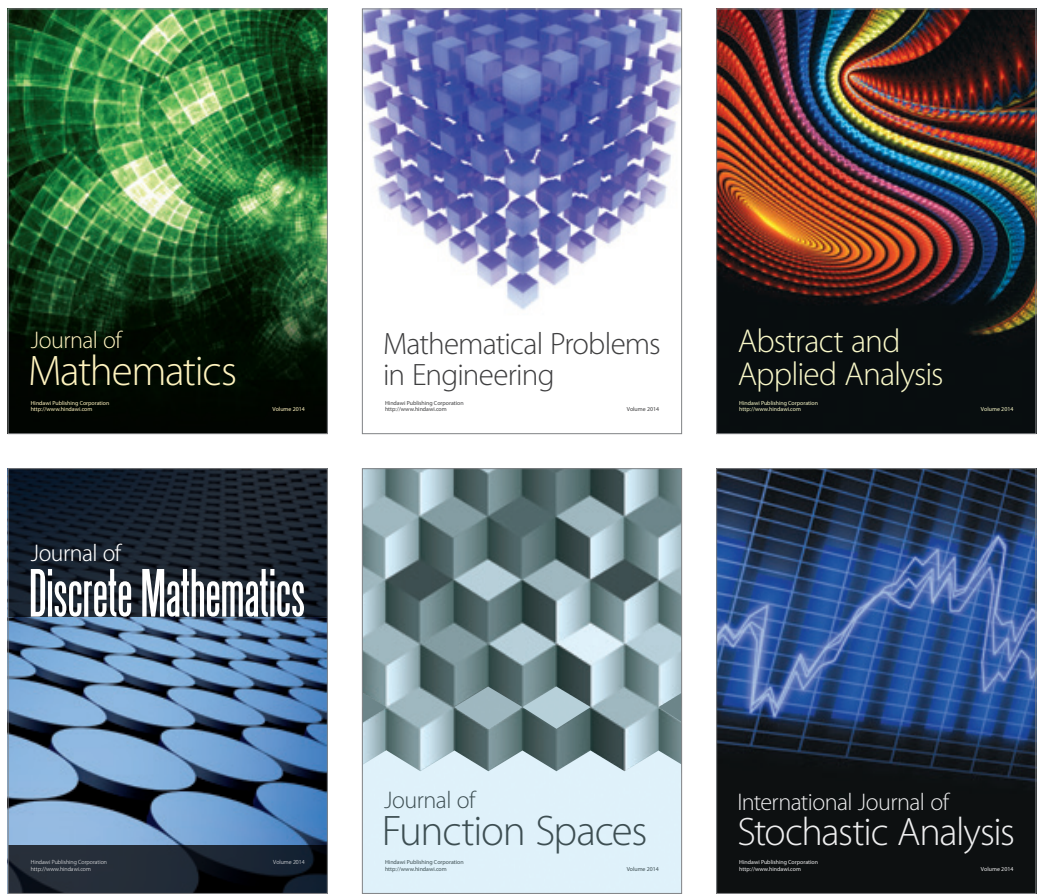

Journal of

Function Spaces

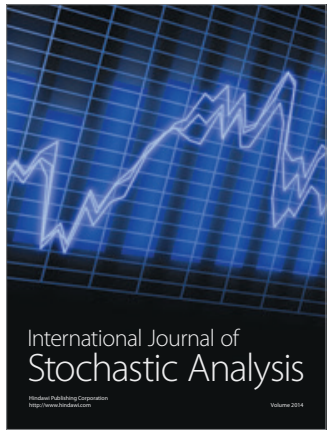

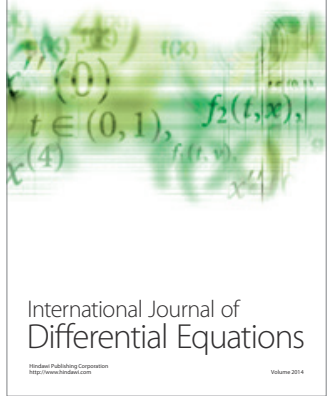
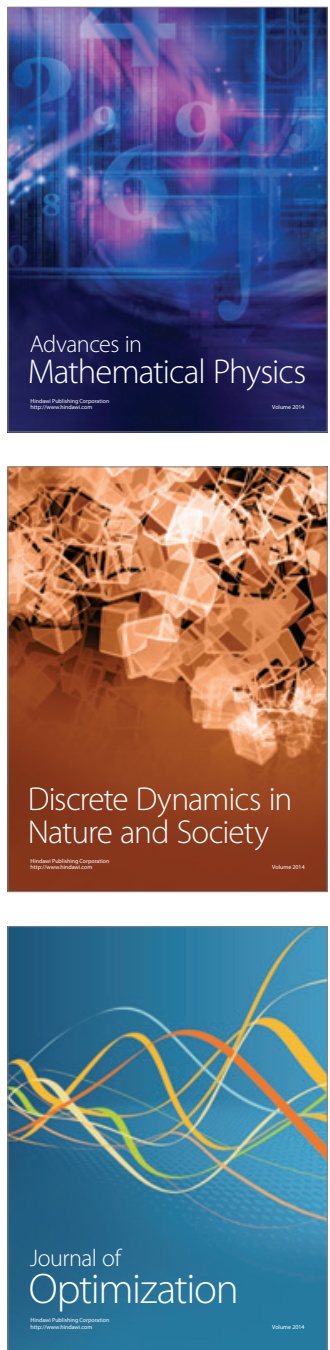